\title{
Limited lymph-node recovery based on lymph-node localisation is sufficient for accurate staging
}

\author{
Gábor Cserni, Rita Bori, István Sejben
}

Bács-Kiskun County Teaching Hospital, Department of Pathology, Kecskemét, Hungary

\section{Correspondence to} Dr Gábor Cserni, Bács-Kiskun County Teaching Hospital, Department of Pathology, Nyiri ut 38., H-6000 Kecskemét, Hungary; cserni@freemail.hu

Accepted 15 September 2010 Published Online First 14 October 2010

\author{
ABSTRACT \\ Aims To assess the reliability of nodal staging in \\ colorectal carcinomas (CRCs) when only lymph nodes \\ close to the tumour are recovered and examined \\ histologically.
}

Methods Lymph nodes from CRC resection specimens were recovered into two fractions: one from around the tumour and the $3 \mathrm{~cm}$ sidelong bowel segment associated with it in the proximal and distal directions, and another from the remaining part of the resection specimen.

Results Of the 762 CRCs (239 right colon, 251 left colon, 257 rectum, 15 unspecified localisation) there were 393 node-negative and 369 node-positive cases. The median number of LNs examined was 18. The assessment of the LNs located in the close fraction (median 13) yielded an adequate qualitative nodal status in 756 patients (99.2\%). In four cases (two rectal and two right colic), no LN metastases could be identified in the close-fraction lymph nodes, but nodes from the distant fraction contained metastases. Of the nodepositive carcinomas which had at least one positive lymph node in the close fraction, 203 belonged to the pN1 category and 162 to the pN2 category of the Tumour Node Metastasis staging system. Only 14 cases (10 rectal, two right and two left colic tumours) were misclassified as pN1 (on the basis of lymph nodes recovered from the close fraction) although they were of the pN2 category.

Conclusions In general, nodal status of CRCs may be adequately assessed by examining the lymph nodes from the close fraction around the tumour and the $3 \mathrm{~cm}$ sidelong bowel segment in both directions.

Lymph node (LN) involvement is an important prognosticator of carcinomas arising in the colon and the rectum. It also influences treatment decisions, as patients with node-positive colorectal carcinoma (CRC) are generally advised to receive some type of systemic adjuvant chemotherapy.

There have been several recommendations regarding the minimum number of $\mathrm{LNs}$ to be assessed for the reliable staging of CRCs. ${ }^{1}$ The most acceptable limit for accurate staging is 12, as suggested by current Tumour Node Metastasisrelated publications. ${ }^{2-4}$ Several papers have also implied a minimum of as few as seven LNs for adequate staging of CRCs. ${ }^{5-7}$ Our previous data also suggested that with fewer than seven LNs assessed, the probability of a false-negative nodal status might be higher. ${ }^{8}$ Suggested minimum numbers generally do not take into consideration that LNs are not equivalent in terms of staging, and some qualitative features may also influence whether accurate staging can be reached with fewer than 12 LNs or not. The size of the LNs is one such possible qualifier, and by taking this into account, we found that the evaluation of the seven largest LNs gives a correct qualitative (negative vs positive) nodal status in $97 \%$ of the cases.

In an earlier study, we evaluated 100 consecutive cases of CRC cases including 53 node-positive cases for the localisation of the LNs assessed in relation to the tumour site. All but one CRCs were correctly staged as node-positive or negative when the LNs from beneath the tumour-involved area and $1 \mathrm{~cm}$ sidelong proximal and distal to it were assessed, and all were correctly staged when the proximal and distal sidelong areas were expanded to $3 \mathrm{~cm}$. It was suggested that correct nodal staging could be reached if all LNs are recovered from the fat around the tumour and the $3 \mathrm{~cm}$ long proximal and distal bowel segment. To improve the accuracy of this policy, the recommendation was supplemented with the removal of additional LNs from the remaining part of the specimen when fewer than seven LNs are assessed (to increase the number of LNs examined) or when there are three LNs involved in the area first assessed (to identify patients more likely to have $\mathrm{pN} 2$ disease). ${ }^{9}$ This study further evaluated the proposed staging practice in a larger series of consecutive patients.

\section{MATERIALS AND METHODS}

All consecutive CRC specimens received between July 2001 and December 2009 were assessed with the intention of dividing the recovered LNs into two fractions: one from the mesocolic or perirectal fat around the tumour and the $3 \mathrm{~cm}$ sidelong bowel segment associated with it in the proximal and distal directions (fraction $\mathrm{AB}$ ) and another from the remaining part of the resection specimen (fraction CD).

Cases where no such division was made (adherence of the tumour involved bowel to other structures preventing correct orientation, difficulty in identifying the tumour site macroscopically and lack of compliance by the pathologist on rotation) were automatically unsuitable for analysis. Patients with multiple or recurrent or intramucosal CRCs, as well as those with no tumour identified after neoadjuvant treatment were excluded.

The length of the bowel and the tumour dimension in this longitudinal projection were recorded after formalin fixation. The distribution of the metastatic LNs was evaluated separately for segments $\mathrm{AB}$ and $\mathrm{CD}$.

For the nodal status, cases were classified according to the pTNM classification of colorectal cancers $^{3} 41011$ into pN0 (with no metastatic LNs), $\mathrm{pN} 1$ (with one to three metastatic LNs) and $\mathrm{pN} 2$ 
(with more than three metastatic LNs) categories. Extramural tumour deposits were not considered metastatic LNs in this study. Cases with isolated tumour cells, that is, tumours with nodal involvement no larger than $0.2 \mathrm{~mm}$, were categorised as $\mathrm{pN} 0$.

\section{RESULTS}

There were 762 CRC cases evaluated in the study. These included 239 cancers in the right colon, 251 in the left colon, 257 in the rectum and 15 carcinomas with unspecified localisation. The mean age of the patients was 66.8 (range 27-94) years. There were 423 male and 339 female patients, and 393 nodenegative and 369 node-positive cancers. The mean number of LNs examined was 19.2 (median 18, range 1-64). The number of LNs examined was lower than seven and 12 in 56 (7.3\%) and $184(24.1 \%)$ cases, respectively.

The assessment of the LNs located in part $A B$ (median 13) of the bowel yielded an adequate qualitative nodal status in 756 patients $(99.2 \%)$. There were two cases where no LNs were recovered from fraction $\mathrm{AB}$ and 24 cases where the bowel length did not allow the separation of a CD fraction. In four (three $\mathrm{pN} 1$, one $\mathrm{pN} 2$; two rectal and two right colic) cases, no LN metastases could be identified in LNs from fraction $\mathrm{AB}$, but LNs from fraction $\mathrm{CD}$ contained metastases (table 1). Of these four cases, three had fewer than seven LNs in segment $\mathrm{AB}$ (two, two and six, respectively) whereas the fourth had 16 negative LNs in part $A B$, and one out of $46 \mathrm{LNs}$ was positive in fraction $\mathrm{CD}$.

Of the node-positive carcinomas which had at least one positive $\mathrm{LN}$ in fraction $\mathrm{AB}, 203$ belonged to the $\mathrm{pN} 1$ category and 162 to the pN2 category. Only 14 cases (10 rectal, two right and two left colic tumours) were misclassified as $\mathrm{pN1}$ (on the basis of LNs recovered from fraction $\mathrm{AB}$ ) although they were of the $\mathrm{pN} 2$ category (on the basis of all LNs); nine of these had three positive LNs, two had two involved nodes, and three had a single metastatic node in the $\mathrm{AB}$ segment.

For the 369 node positive CRCs, the rate of metastatic AB LNs was $1549 / 5317$ (29.1\%; 95\% CI $27.9 \%$ to $30.4 \%$ ), whereas the rate of metastatic CD lymph nodes was only 155/2041 (7.6\%; $95 \%$ CI $6.4 \%$ to $8.7 \%)$, the difference being highly significant $\left(\chi^{2}\right.$ $383.28, \mathrm{p}<0.0001)$.

There were 17 cases with isolated tumour cells as the most significant nodal finding. As stated in the Materials and methods, these were put into the $\mathrm{pN} 0$ category, but were also looked at separately: 16 of these cases had their nodal involvement in LNs from fraction $\mathrm{AB}$, and one case of rectal carcinoma assessed after neoadjuvant therapy had only involvement of an LN from fraction CD.

With the original approach suggested in our preliminary work and quoted at the end of the introduction, ${ }^{9}$ only one out of 762 cases would have been falsely classified as node-negative, which would mean $99.9 \%$ accuracy and a $0.3 \%$ false-negative rate (one false-negative case per 369 node-positive cases). The same would have applied if cases with fewer than 12 (and not seven) nodes in part $A B$ had been assessed for further LNs from segments $C D$.

Table 1 Distribution of node-negative and node-positive colorectal carcinoma cases according to the localisation of the lymph nodes

\begin{tabular}{lcrcr}
\hline Fraction AB/fraction CD & $\begin{array}{l}\text { Not applicable } \\
\text { (NA) }\end{array}$ & pN0 & pN1/pN2 & All \\
\hline NA & 0 & 2 & 0 & 2 \\
pN0 & 13 & 377 & 4 & 394 \\
pN1/pN2 & 11 & 294 & 61 & 366 \\
All & 24 & 673 & 65 & 762 \\
\hline
\end{tabular}

For a more detailed $\mathrm{pN}$ category determination, the original approach would have missed the above case ( $\mathrm{pN} 1$ classified as $\mathrm{pN} 0$ ) and would have wrongly classified three $\mathrm{pN} 2$ cases as $\mathrm{pN} 1$; the remaining 11 cases with fewer than four involved $A B$ LNs would have been correctly identified as $\mathrm{pN} 2$ due to the low number of $A B$ nodes $(n=2)$, the presence of three metastatic $A B$ nodes $(n=5)$ or both of these $(n=4)$. This would have resulted in a $\mathrm{pN}$ categorisation accuracy of $99.5 \%$. Raising the limit of the minimum number of LNs required for examination (in segment $A B)$ from seven to 12 would have resulted in only one $p N 2$ case wrongly labelled as pN1 (2/14 AB nodes positive and $2 / 3 \mathrm{CD}$ nodes positive) and a $\mathrm{pN}$ categorisation accuracy of $99.7 \%$.

\section{DISCUSSION}

There have been several recommendations regarding the minimum number of $\mathrm{LNs}$ to be assessed for the reliable staging of $\mathrm{CRCs}^{1}$ ranging from $6^{12}$ to $20,{ }^{13}$ although it is mostly accepted that there is no such a minimum, and all LNs need to be recovered from resection specimens and evaluated by histology. The staging accuracy increases with the number of LNs assessed, and such an improvement in staging reliability is also associated with improved survival. ${ }^{14} 15$ In the setting recommending the examination of all LNs resected, the suggested values for the number of LNs to be assessed are better understood as numbers reflecting the accuracy of staging. These numerical recommendations never took into account the fact that LNs are not equivalent in terms of target for metastatic spread, and qualitative features may influence the accuracy of staging, even when fewer than all LNs resected are examined histologically.

Several qualitative features of the LNs enabling a selection between metastatic and metastasis-free ones have been assessed, including their size, ${ }^{7}$ their localisation ${ }^{9}$ and their direct connection with the primary tumour site assessed by lymphatic mapping. ${ }^{81617}$ On the basis of a preliminary study, based on the analysis of 100 CRC cases, we have proposed that metastatic LNs (at least the first ones) are more likely to be located in the close vicinity of the tumour. It was concluded that this close vicinity was the mesocolon/mesorectum beneath the length of resected bowel including the tumour and the $3 \mathrm{~cm}$ sidelong part proximal and/or distal to it. Our present data strengthen this conclusion, as they make it clear that analysing the LNs in this part of the pericolic and perirectal fat (segment $A B$ ) is sufficient for the accurate staging of CRCs as node-positive or negative in nearly all cases, and it also allows an accurate $\mathrm{pN} 0$ versus $\mathrm{pN} 1$ versus $\mathrm{pN} 2$ classification in most cases. Whenever $\mathrm{LNs}$ in segment $A B$ are involved, the chances of having further metastatic LNs in the rest of the nodes are increased. Indeed, 61 of the 365 CRCs (16.7\%) with positive $A B$ nodes had nodal involvement in CD LNs, too. Adding some further precaution to avoid misclassification such as the examination of CD LNs when there are three $A B$ nodes involved or when the number of $A B L N$ is low (fewer than seven in our original recommendation, or fewer than 12 to comply with most recommendations) should even improve the staging accuracy with limited workload.

Besides our initial report, ${ }^{9}$ we are aware of only one study which assessed the localisation of LNs in a fashion similar to ours. Pusztaszeri et al, in a recent study of 345 CRCs, used a cutoff length of $5 \mathrm{~cm}$ in both directions from the tumour and concluded that metastases seldom occur in the distant nodes only. ${ }^{18}$ They reported a just lower median number of LNs overall $(n=17)$ and noted only six cases which were devoid of metastatic LNs in the close fraction but had positive nodes in the distant fraction. Although their accuracy rate for a qualitative 


\section{Take-home messages}

Lymph nodes (LNs) are not equivalent in terms of target for metastatic spread.

- Metastastic LNs almost never occur in distant locations from the tumour if no close LNs are involved.

nodal status was somewhat below ours (98.3\%), this is not a major difference. Most of their false-negative statuses (five out of six) were in rectal cancers where they recommended the examination of all LNs. Our findings do not fully match this distribution, but half of the false-negative cases (2/4) and most $(10 / 14)$ of the understaged tumours were in the rectum, a fact that may support a more intensive search for LNs if the primary cancer is in this region.

Although it has been suggested that sentinel LNs of CRCs may be located away from the primary tumour and can be found even at sites falling outside their traditional resection margins (eg, at the splenic flexure for a carcinoma in the ascendent colon), ${ }^{19}{ }^{20}$ this is a rare phenomenon (Sukamal Saha, oral communication at the 5th East-West Colorectal Days, Hajduszoboszlo, 10-12 September 2009). The rarity of such distant nodal metastases is also supported by the present data. It must also be considered that the pooled false-negative rate of sentinel LN examination in CRCs is around $9 \%$, ${ }^{17}$ which is much higher than the false-negative rate of assessing only the LNs close to the primary tumour as defined in the present study.

It is suggested that sufficiently accurate nodal staging can be reached by the limited and stepwise protocol we suggest (ie, the assessment of relatively distant LNs only if necessary either because of a higher suspicion for a higher nodal category or because of too few LNs recovered during the limited work-up). This may spare time and reduce workload in case of long resected segments such as hemicolectomy specimens. As most LNs are recovered from the area we propose to investigate first, the number of nodes assessed should also not diminish too much with the recommended approach. However, the present results should not be used to substantiate substandard $\mathrm{LN}$ recovery rates potentially leading to understaging, and all LNs in the suggested area should be thoroughly looked for.
Competing interests None.

Provenance and peer review Not commissioned; externally peer reviewed.

\section{REFERENCES}

1. Cserni G. Nodal staging of colorectal carcinomas and sentinel nodes. J Clin Pathol 2003:56:327-35.

2. Wittekind Ch, Greene FL, Henson DE, et al, eds. TNM Supplement-A Commentary on Uniform Use. 3rd edn. New York: John Wiley and Sons, 2003.

3. Greene FL, Page DL, Morrow M, et al, eds. AJCC Cancer Staging Manual. 6th edn. New York: Springer, 2002

4. Edge SB, Byrd DR, Compton CC, et al, eds. AJCC Cancer Staging Handbook: From the AJCC Cancer Staging Manual. 7th edn. New York: Springer, 2009.

5. Caplin S, Cerottini J-P, Bosman FT, et al. For patients with Dukes' B (TNM stage II) colorectal carcinoma, examination of six or fewer lymph nodes is related to poor prognosis. Cancer 1998;83:666-72.

6. Mainprize KS, Kulacoglu $\mathrm{H}$, Hewavisinthe J, et al. How many lymph nodes to stage colorectal carcinomas? J Clin Pathol 1998;51:165-6.

7. Cserni G. The influence of nodal size on the staging of colorectal carcinomas. J Clin Pathol 2002;55:386-90.

8. Cserni G, Vajda K, Tarján M, et al. Nodal staging of colorectal carcinomas from quantitative and qualitative aspects. Can lymphatic mapping help staging? Pathol Oncol Res 1999:5:291-6.

9. Cserni G, Tarján M, Bori R. Distance of lymph nodes from the tumor, an important feature in colorectal cancer specimens. Arch Pathol Lab Med 2001;125:246-9.

10. Sobin LH, Wittekind Ch, eds. UICC TNM Classification of Malignant Tumours. 6th edn. New York: John Wiley and Sons, 2002.

11. Sobin L, Gospodarowicz M, Wittekind Ch, eds. UICC TNM Classification of Malignant Tumours. 7th edn. New York: John Wiley and Sons, 2009.

12. Hernanz F, Revuelta S, Redondo C, et al. Colorectal adenocarcinoma: quality of the assessment of lymph node metastases. Dis Colon Rectum 1994:37:373-6.

13. Greco P, Andreola S, Magro G, et al. Potential pathological understaging of pT3 rectal cancer with less than 26 lymph nodes recovered: a prospective study based on a resampling of 50 rectal specimens. Virchows Arch 2006;449:647-51.

14. Goldstein NS. Lymph node recoveries from 2427 pT3 colorectal resection specimens spanning 45 years: recommendations for a minimum number of recovered lymph nodes based on predictive probabilities. Am J Surg Pathol 2002;26:179-89.

15. Cserni G, Vinh-Hung V, Burzykowski T. Is there a minimum number of lymph nodes that should be histologically assessed for a reliable nodal staging of T3NOMO colorectal carcinomas? J Surg Oncol 2002;81:63-9.

16. Saha S, Wiese D, Badin J, et al. Technical details of sentinel lymph node mapping in colorectal cancer and its impact on staging. Ann Surg Oncol 2000;7:120-4.

17. Des Guetz G, Uzzan B, Nicolas P, et al. Is sentinel lymph node mapping in colorectal cancer a future prognostic factor? A meta-analysis. World J Surg 2007;31:1304-12.

18. Pusztaszeri M, Matter M, Kuonen $\mathbf{M}$, et al. Nodal staging in colorectal cancer: should distant lymph nodes be recovered in surgical specimens? Hum Pathol 2009:40:552-7.

19. Tsoulias GJ, Wood TF, Morton DL, et al. Lymphatic mapping and focused analysis of sentinel lymph nodes upstage gastrointestinal neoplasms. Arch Surg 2000;135:926-32.

20. Bilchik AJ, Saha S, Wiese D, et al. Molecular staging of early colon cancer on the basis of sentinel node analysis: a multicenter phase II trial. J Clin Oncol 2001;19:1128-36. 